\title{
Hydrocortisone Reduces 28-day Mortality in Septic Patients: A Systemic Review and Meta- analysis
}

Waqas J. Siddiqui ${ }^{1,2}$, Praneet Iyer ${ }^{3}$, Ghulam Aftab ${ }^{4}$, FNU Zafrullah ${ }^{5}$, Muhammad Zain ${ }^{6}$, Kadambari Jethwani ${ }^{7}$, Rabia Mazhar ${ }^{8}$, Usman Abdulsalam ${ }^{9}$, Abbas Raza ${ }^{7}$, Muhammad O. Hanif ${ }^{10,7}$, Esha Sharma ${ }^{11}$ , Sandeep Aggarwal 10

1. Cardiology/Nephrology, Drexel University College of Medicine, Philadelphia, USA 2. Cardiology/Nephrology, Orange Park Medical Center, Orange Park, USA 3. Internal Medicine, University of Tennessee Health Sciences Center, Memphis, USA 4. Pulmonary Medicine, St. Peter's University Hospital, New Brunswick, USA 5. Internal Medicine, Steward Carney Hospital, Tufts University School of Medicine, Boston, USA 6. Internal Medicine, Sheikh Zayed Medical College and Hospital, Rahim Yar Khan, PAK 7. Internal Medicine, Drexel University, Philadelphia, USA 8. Internal Medicine, Orange Park Medical Center, Orange Park, USA 9. Internal Medicine, Steward Carney Hospital, Boston, USA 10. Nephrology, Drexel University, Philadelphia, USA 11. Internal Medicine, George Washington University, Washington D.C., USA

Corresponding author: Ghulam Aftab, gaftab@saintpetersuh.com

\begin{abstract}
The goal of this study was to determine the utility of hydrocortisone in septic shock and its effect on mortality. We performed a systematic search from inception until March 01, 2018, according to PRISMA (Preferred Reporting Items for Systematic Reviews and Meta-Analyses) guidelines comparing hydrocortisone to placebo in septic shock patients and selected studies according to our pre-defined inclusion and exclusion criteria. Four reviewers extracted data into the predefined tables in the Microsoft Excel (Microsoft Corp., New Mexico, US) sheet. We used RevMan software to perform a meta-analysis and draw Forest plots. We used a random effects model to estimate risk ratios. A two-sided $p$-value of $\leqslant 0.05$ was considered statistically significant. A total of five randomized control trials (RCTs) with 5,838 patients were included in our analysis. The primary outcome was mortality at 28 days. Secondary outcomes were intensive care unit (ICU) and in-hospital mortality, mortality at 90 days and one year, reversal of shock, intensive care unit (ICU) and hospital length of stay, incidence of superinfections, and incidence of limb and/or cerebral ischemia. The 28-day mortality was significantly reduced with hydrocortisone, 808 vs. 880 with placebo, Risk Ratio $(\mathrm{RR})=0.92$, confidence interval $(\mathrm{CI})=0.85-0.99, \mathrm{p}=0.04, \mathrm{I}^{2}=0 \%$. There was no difference in ICU mortality ( $\mathrm{RR}=0.93$, $\mathrm{CI}=0.81-1.08)$, in-hospital mortality $(\mathrm{RR}=0.95, \mathrm{CI}=0.84-1.08)$, 90-day mortality $(\mathrm{RR}=0.93, \mathrm{CI}=0.84-1.02, \mathrm{p}=0.10)$, and one-year mortality $(\mathrm{RR}=0.97, \mathrm{CI}=0.84-1.12)$. Superinfections were significantly common with hydrocortisone, $\mathrm{RR}=1.16, \mathrm{CI}=1.05-1.28, \mathrm{p}=0.003$. In conclusion, the use of hydrocortisone showed a significant reduction in mortality at 28 days and a trend toward reduced ICU mortality. This mortality reduction was observed at the cost of significantly higher superinfections.
\end{abstract}

Received 04/22/2019 Review began 05/23/2019 Review ended 06/07/2019 Published 06/17/2019

(๑) Copyright 2019

Siddiqui et al. This is an open access article distributed under the terms of the Creative Commons Attribution License CC-BY 3.0. which permits unrestricted use, distribution, and reproduction in any medium, provided the original author and source are credited.
Categories: Internal Medicine, Infectious Disease, Other

Keywords: hydrocortisone, sepsis, septic shock, fludrocortisone, mortality, meta-analysis

\section{Introduction}

Sepsis is a significant health concern globally with an associated mortality of $14.7 \%$ to $29.9 \%$ [1]. Over the years, although the number of sepsis cases and total mortality has increased, the associated case fatality rate has decreased [1]. Other than the respiratory support with mechanical ventilation, hemodynamic support with fluid resuscitation and vasopressors, and source control of the infection with antibiotics and surgical evacuation of infection, there is no additional approved treatment for either sepsis or septic shock

[2]. Steroids have been evaluated as a treatment option for several years. The suggested theory behind the use of steroids is that they suppress inflammatory mediators and treat sepsis-induced relative adrenal insufficiency, which has been studied in various trials and is yet to be proved [3-4].

A study from 1976 by Schumer et al. showed a significant reduction in mortality in septic shock with high dose steroids given for a short duration [5]. However, several subsequent studies were unable to replicate these findings but, in turn, showed increased associated mortality due to a higher incidence of superinfection, defined as a new clinical infection that occurred during therapy or within ten days of discontinuation of antimicrobial agents [6-8]. The use of steroids was discouraged until 2002 when the French study by Annane et al. showed significant mortality benefit with the use of steroids, which brought steroids back in favor [9]. Several subsequent studies, including systematic reviews, meta-analysis, and randomized control trials (RCTs), have not shown consistent evidence for or against steroids in sepsis and septic shock [10-12]. Current surviving sepsis guidelines recommend the use of steroids when fluid resuscitation and vasopressors are not effective in correcting hemodynamic instability, but this remains a weak recommendation [2]. The two recent RCTs evaluating the use of hydrocortisone in septic shock patients suggested conflicting results leaving clinicians with no explicit guidance [13-14]. There have been 
no meta-analysis to date which only utilized RCTs for systematic review and meta-analysis. The earlier systematic reviews have used studies, which had a variable patient population with systemic inflammatory response syndrome, sepsis, or septic shock. Those meta-analysis included RCTs, non-randomized trials, observational retrospective, and prospective studies and studies from the 1980s and 1990s where they also used dexamethasone, prednisone, and methylprednisone instead of only potent mineral corticoids, which are hydrocortisone and fludrocortisone. They also included studies in which steroids were used for reasons other than septic shock (e.g., meningitis). Our meta-analysis only incorporates RCTs, which included patients only with septic shock and utilized hydrocortisone alone or with fludrocortisone. Recently published RCTs in The New England Journal of Medicine (NEJM) on March 1, 2018, have not been part of any of the prior systematic reviews and meta-analyses, and the editorial published in the same edition of NEJM recommended to decide about patient management on the basis of subsequent meta-analyses utilizing these two RCTs [15].

\section{Materials And Methods}

We conducted this meta-analysis is to identify the effect of potent mineralocorticoids (hydrocortisone and fludrocortisone) in refractory septic shock patients with possible underlying relative adrenal and mineral corticoid insufficiency and their impact on short-term (defined as 28-day) mortality. The other steroids lack significant mineral corticoid activity as compared to hydrocortisone and fludrocortisone. For the primary outcome, we also performed the sub-group analysis by time to administration of hydrocortisone from time to randomization (early, i.e., within eight hours of randomization vs. late, i.e., within 24-72 hours of randomization). Our secondary outcomes assessed the long-term survival with the intensive care unit (ICU) and the hospital mortality and length of stay (LOS) with reversal of shock. We also evaluated the difference in commonly encountered complications of septic shock, including the incidence of superinfections and limb and cerebral ischemic events.

We completed a systematic review according to the PRISMA (Preferred Reporting Items for Systematic Review and Meta-Analyses) guidelines [16]. We searched the MEDLINE and PubMed databases from inception until March 01, 2018, only for RCTs, comparing the use of hydrocortisone to the placebo in septic shock patients.

Our search strategy included (glucocorticoid OR hydrocortisone OR steroid) AND (sepsis OR septic OR septic shock). We used the Boolean operator 'OR' to combine the search terms.

\section{Inclusion criteria}

1) Prospective RCTs, 2) Comparing hydrocortisone with or without fludrocortisone to placebos in patients with documented septic shock, 3) Patients age $\geqslant 18$ years, 4) At least 100 patients were randomized in the study, and 5) At least one endpoint was 28-day mortality.

\section{Exclusion criteria}

We excluded non-randomized and retrospective studies, studies which used steroids other than hydrocortisone, and total number of patients was $<100$; studies that included patients with sepsis and severe sepsis, were in the non-English language, lacked 28-day mortality data, and in which both arms received hydrocortisone.

\section{Primary endpoints}

The primary endpoint was mortality at 28 days.

\section{Secondary endpoints}

We analyzed the following secondary endpoints: 1) Mortality in ICU; 2) Mortality in the hospital; 3) Mortality at 90 days; 4) Mortality at one year; 5) Reversal of Shock; 6) ICU LOS; 7) Hospital LOS; 8) Incidence of limb and/or cerebral ischemia; and 9) Incidence of superinfection.

\section{Data extraction and quality assessment}

Four reviewers, W.J.S., A.R., U.A.S., and M.O.H. extracted the data in the predefined data fields in the Excel sheet for baseline characteristics and study outcomes. They added outcomes that were mentioned in the outcomes tables and described in the text. W.J.S. cross-checked all the entered data and made corrections where necessary. All four reviewers agreed with the corrections and the final entry. Table 1 shows the features and differences of individual RCTs, and Table 2 summarizes the baseline characteristics of individual trials $[9,11-14,17]$. We used Cochrane collaboration's tool risk assessment of bias in randomized trials for the quality assessment of RCTs [18] (Figures 1-2 and Table 3). 


\section{Cureus}

\begin{tabular}{|c|c|c|c|c|}
\hline Outcome & Effect Estimate & Confidence Interval & p-value & $I^{2}(\%)$ \\
\hline \multicolumn{5}{|l|}{ Primary Outcome } \\
\hline Mortality at 28 days & 0.92 & $0.85-0.99$ & 0.04 & 0 \\
\hline \multicolumn{5}{|l|}{ Secondary Outcomes } \\
\hline Mortality in Intensive Care Unit After sensitivity analysis & 0.930 .87 & $0.81-1.080 .78-0.97$ & 0.350 .01 & 520 \\
\hline Mortality in the hospital & 0.95 & $0.84-1.08$ & 0.41 & 39 \\
\hline Mortality at 90 days & 0.93 & $0.84-1.02$ & 0.13 & 37 \\
\hline Mortality at one year & 0.97 & $0.84-1.12$ & 0.67 & 46 \\
\hline Reversal of Shock & 1.17 & $0.74-1.86$ & 0.5 & 24 \\
\hline Intensive Care Unit Length of Stay & 0.89 & -2.56 to 4.33 & 0.61 & 0 \\
\hline Hospital Length of Stay & 1.58 & -4.23 to 7.38 & 0.59 & 0 \\
\hline The incidence of Superinfection & 1.15 & $1.04-1.27$ & 0.008 & 0 \\
\hline The incidence of limb and/or cerebral ischemia & 1.32 & $0.30-5.90$ & 0.72 & 0 \\
\hline
\end{tabular}

TABLE 1: Outcomes 


\section{Cureus}

\begin{tabular}{|c|c|c|c|c|c|c|c|c|c|c|c|}
\hline Name & Design & Country & $\begin{array}{l}\text { Publication } \\
\text { Year }\end{array}$ & Journal & Enrollment & Population & $\begin{array}{l}\text { Time to } \\
\text { randomization } \\
\text { from the } \\
\text { onset of } \\
\text { shock }\end{array}$ & Setting & $\begin{array}{l}\text { Intervention } \\
\text { Vs. } \\
\text { Comparison }\end{array}$ & $\begin{array}{l}\text { Dose and } \\
\text { Type of } \\
\text { Steroid and } \\
\text { Route of } \\
\text { Administration }\end{array}$ & $\begin{array}{l}\text { F/u } \\
\text { Duration }\end{array}$ \\
\hline $\begin{array}{l}2018 \\
\text { APROCCHSS } \\
\text { trial [14] }\end{array}$ & $\begin{array}{l}\text { Double- } \\
\text { blind } \\
\text { placebo- } \\
\text { controlled } \\
\text { RCT }\end{array}$ & France & $3 / 1 / 18$ & NEJM & $\begin{array}{l}\text { September } \\
\text { 2008- June } \\
2015\end{array}$ & $\begin{array}{l}\text { Septic } \\
\text { shock }\end{array}$ & $\begin{array}{l}\text { Within } 24 \\
\text { hours of onset } \\
\text { of shock }\end{array}$ & ICU & $\begin{array}{l}\text { Hydrocortisone } \\
\text { plus } \\
\text { Fludrocortisone } \\
\text { vs. Placebo }\end{array}$ & $\begin{array}{l}50 \text { mg IV Q6 } \\
\text { hours plus } 50 \\
\mu g \text { 9- } \alpha- \\
\text { fludrocortisone } \\
\text { via NG tube for } \\
7 \text { days w/o } \\
\text { tapering }\end{array}$ & 180 days \\
\hline $\begin{array}{l}2018 \\
\text { ADRENAL } \\
\text { trial [13] }\end{array}$ & $\begin{array}{l}\text { Double- } \\
\text { blind } \\
\text { placebo- } \\
\text { controlled } \\
\text { RCT }\end{array}$ & $\begin{array}{l}\text { UK, NZ, } \\
\text { KSA, } \\
\text { Australia, } \\
\text { Denmark }\end{array}$ & 1/19/18 & NEJM & $\begin{array}{l}\text { March } \\
2013-\text { April } \\
2017\end{array}$ & $\begin{array}{l}\text { Septic } \\
\text { shock }\end{array}$ & $\begin{array}{l}\text { Within } 24 \\
\text { hours of onset } \\
\text { of shock }\end{array}$ & ICU & $\begin{array}{l}\text { Hydrocortisone } \\
\text { vs. Placebo }\end{array}$ & $\begin{array}{l}200 \mathrm{mg} / \mathrm{d} \text { as a } \\
\text { continuous IV } \\
\text { Infusion for } 7 \\
\text { days }\end{array}$ & 90 days \\
\hline $\begin{array}{l}2017 \text { Qing- } \\
\text { quan Lv et al. } \\
\text { [11] }\end{array}$ & $\begin{array}{l}\text { Double- } \\
\text { blind } \\
\text { placebo- } \\
\text { controlled } \\
\text { RCT }\end{array}$ & China & $6 / 4 / 17$ & AJEM & $\begin{array}{l}\text { September } \\
2015 \text { - } \\
\text { September } \\
2016\end{array}$ & $\begin{array}{l}\text { Septic } \\
\text { shock }\end{array}$ & $\begin{array}{l}\text { Within } 6 \text { hours } \\
\text { of onset of } \\
\text { shock }\end{array}$ & ICU & $\begin{array}{l}\text { Hydrocortisone } \\
\text { vs. Placebo }\end{array}$ & $\begin{array}{l}200 \mathrm{mg} / \mathrm{d} \text { as a } \\
\text { continuous IV } \\
\text { Infusion for } 6 \\
\text { days, then } \\
\text { tapered during } \\
\text { a 6-day period }\end{array}$ & 28 days \\
\hline $\begin{array}{l}2008 \\
\text { CORTICUS } \\
\text { trial [17] }\end{array}$ & $\begin{array}{l}\text { Double- } \\
\text { blind } \\
\text { placebo- } \\
\text { controlled } \\
\text { RCT }\end{array}$ & $\begin{array}{l}\text { Austria, } \\
\text { Israel, } \\
\text { Belgium, } \\
\text { UK, } \\
\text { Germany, } \\
\text { France, } \\
\text { Portugal, } \\
\text { Netherlands }\end{array}$ & 1/10/08 & NEJM & $\begin{array}{l}\text { March } \\
2002- \\
\text { November } \\
2005\end{array}$ & $\begin{array}{l}\text { Septic } \\
\text { shock }\end{array}$ & $\begin{array}{l}\text { Within } 72 \\
\text { hours of onset } \\
\text { of shock }\end{array}$ & ICU & $\begin{array}{l}\text { Hydrocortisone } \\
\text { vs. Placebo }\end{array}$ & $\begin{array}{l}50 \mathrm{mg} \text { IV Q6 } \\
\text { hours for } 5 \\
\text { days; then } \\
\text { tapered during } \\
\text { a 6-day period }\end{array}$ & 28 days \\
\hline $\begin{array}{l}2002 \text { Annane } \\
\text { et al. [9] }\end{array}$ & $\begin{array}{l}\text { Double- } \\
\text { blind } \\
\text { placebo- } \\
\text { controlled } \\
\text { RCT }\end{array}$ & France & 8/21/02 & JAMA & $\begin{array}{l}\text { September } \\
1995 \text { - } \\
\text { March } \\
1999\end{array}$ & $\begin{array}{l}\text { Septic } \\
\text { shock }\end{array}$ & $\begin{array}{l}\text { Within } 8 \text { hours } \\
\text { of onset of } \\
\text { shock }\end{array}$ & ICU & $\begin{array}{l}\text { Hydrocortisone } \\
\text { plus } \\
\text { Fludrocortisone } \\
\text { vs. Placebo }\end{array}$ & $\begin{array}{l}50 \text { mg IV Q6 } \\
\text { hours plus } 50 \\
\mu g \text { 9- } \alpha- \\
\text { fludrocortisone } \\
\text { via NG tube for } \\
7 \text { days w/o } \\
\text { tapering }\end{array}$ & 28 days \\
\hline
\end{tabular}

\section{TABLE 2: Characteristics of Randomized Control Trials}

$\mathrm{RCT}=$ Randomized Control Trial, F/u = Follow up, $\mathrm{n} .=$ number, NEJM $=$ New England Journal of Medicine AJEM: American Journal of Emergency Medicine. JAMA: Journal of the American Medical Association, NZ: New Zealand, KSA: Kingdom of Saudi Arabia, UK: United Kingdom; ICU: Intensive Care Unit, IV = intravenous, w/o = without 


\section{Cureus}

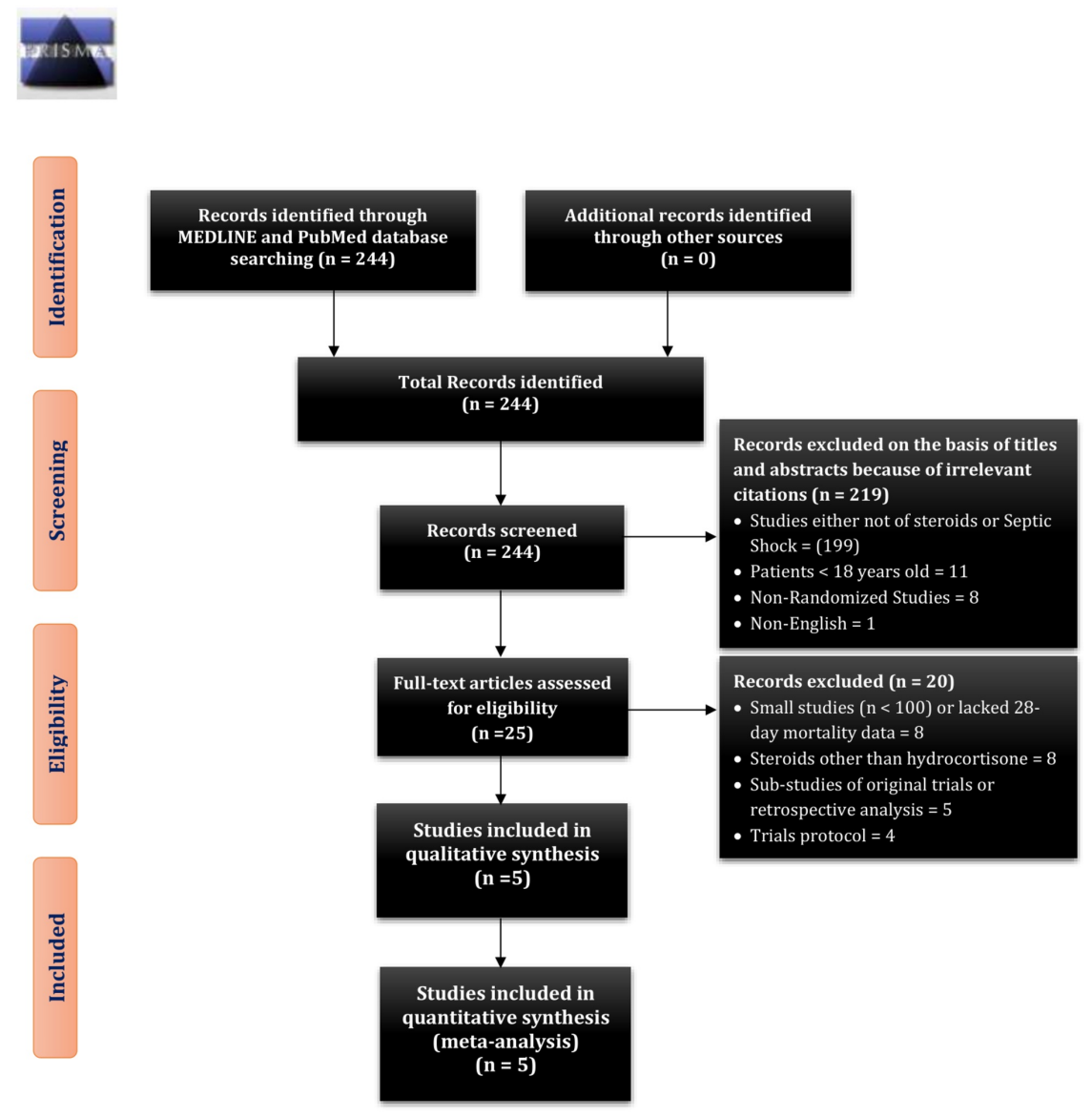

FIGURE 1: PRISMA 2009 STUDY SEARCH AND SELECTION DIAGRAM

PRISMA = Preferred Reporting Items for Systematic Reviews and Meta-analyses

FIGURE 1: PRISMA 2009 Study Search and Selection Diagram

PRISMA: Preferred Reporting Items for Systemic Reviews and Meta-analyses

Figure 2 Primary Outcome Mortality at 28-Days

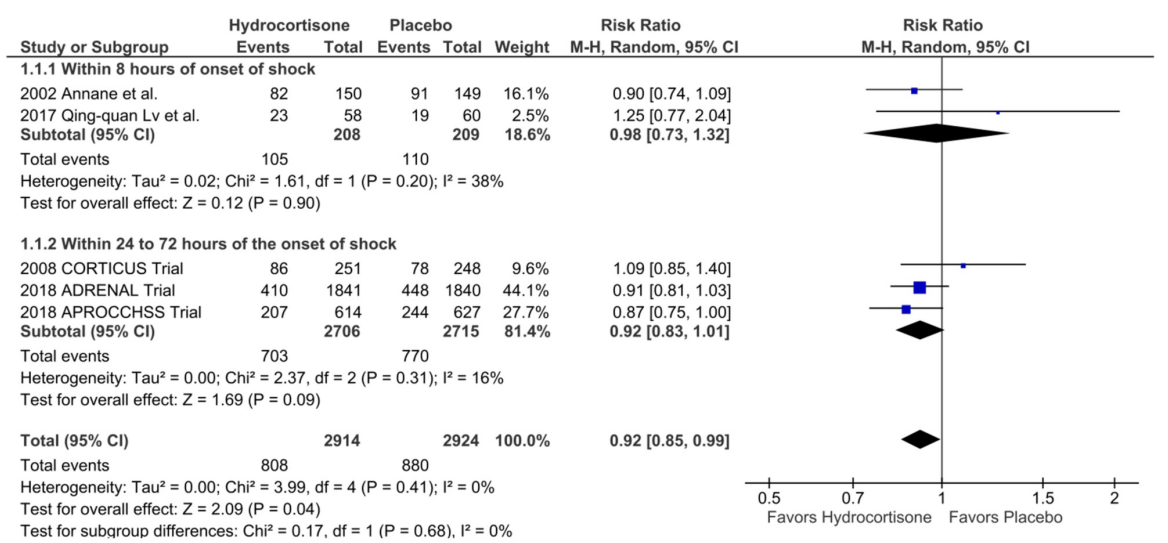

FIGURE 2: Primary Outcome - Mortality at 28 Days 


\section{Cureus}

\begin{tabular}{|c|c|c|c|c|c|c|c|c|c|c|}
\hline Studies & $\begin{array}{l}2018 \\
\text { APROCCHSS }\end{array}$ & $\begin{array}{l}2018 \\
\text { ADRENAL }\end{array}$ & $\begin{array}{l}2017 \text { Qing- } \\
\text { quan Lv et al. }\end{array}$ & $\begin{array}{l}2008 \\
\text { CORTICUS }\end{array}$ & \multicolumn{2}{|c|}{2002 Annane et al. [9] } & & \multirow[b]{2}{*}{ Placebo } & & \multirow[b]{2}{*}{ Placebo } \\
\hline Treatment arms & $\begin{array}{l}\text { Hydrocortisone } \\
+ \\
\text { Fludrocortisone }\end{array}$ & Placebo & Hydrocortisone & Placebo & Hydrocortisone & Placebo & Hydrocortisone & & $\begin{array}{l}\text { Hydrocortisone } \\
+ \\
\text { Fludrocortisone }\end{array}$ & \\
\hline $\mathrm{N}$ & 614 & 627 & 1853 & 1860 & 58 & 60 & 251 & 248 & 150 & 149 \\
\hline $\begin{array}{l}\text { Male sex - no. } \\
(\%)\end{array}$ & $402(65.5)$ & $424(67.7)$ & $1119(60.4)$ & $1140(61.3)$ & $33(56.9)$ & $37(61.7)$ & $166(66)$ & $166(67)$ & $96(64)$ & 104(70) \\
\hline $\begin{array}{l}\text { Age }- \text { years } \\
\text { Mean } \pm S D\end{array}$ & $66 \pm 14$ & $66 \pm 15$ & $62.3 \pm 14.9$ & $62.7 \pm 15.2$ & $68.8 \pm 12.6$ & $64.8 \pm 16.7$ & $63 \pm 14$ & $63 \pm 15$ & $62(15)$ & $60(17)$ \\
\hline $\begin{array}{l}\text { Whites - no. } \\
(\%)\end{array}$ & N/A & $\mathrm{N} / \mathrm{A}$ & $\mathrm{N} / \mathrm{A}$ & $\mathrm{N} / \mathrm{A}$ & N/A & N/A & $236(94)$ & $228(92)$ & $137(92)$ & $139(95)$ \\
\hline $\begin{array}{l}\text { Admissions } \\
\text { from Medical } \\
\text { Ward no. (\%) }\end{array}$ & $495(82.4)$ & $499(81)$ & $1273(68.8)$ & $1266(68.2)$ & $17(41.5)$ & $22(57.9)$ & $80(32)$ & $93(38)$ & $89(59)$ & $90(60)$ \\
\hline $\begin{array}{l}\text { Admissions } \\
\text { from Surgery } \\
\text { No. }(\%)\end{array}$ & N/A & $\mathrm{N} / \mathrm{A}$ & $576(31.2)$ & $591(31.8)$ & N/A & $\mathrm{N} / \mathrm{A}$ & $169(67.8)$ & $153(62)$ & $61(40.7)$ & $59(39.6)$ \\
\hline SAPS II & $56 \pm 19$ & $56 \pm 19$ & $\mathrm{~N} / \mathrm{A}$ & $\mathrm{N} / \mathrm{A}$ & $\mathrm{N} / \mathrm{A}$ & $\mathrm{N} / \mathrm{A}$ & $49.5 \pm 17.8$ & $48.6 \pm 16.7$ & $60(19)$ & $57(19)$ \\
\hline SAPS III & $\mathrm{N} / \mathrm{A}$ & N/A & $\mathrm{N} / \mathrm{A}$ & $\mathrm{N} / \mathrm{A}$ & N/A & N/A & N/A & N/A & $\mathrm{N} / \mathrm{A}$ & N/A \\
\hline $\begin{array}{l}\text { APACHE II } \\
\text { Score Mean } \pm \\
\text { SD }\end{array}$ & N/A & $\mathrm{N} / \mathrm{A}$ & 24 & 23 & $25.5 \pm 9.5$ & $21.3 \pm 6.9$ & N/A & $N / A$ & N/A & N/A \\
\hline $\begin{array}{l}\text { SOFA Score } \\
\text { Mean } \pm \text { SD }\end{array}$ & $12 \pm 3$ & $11 \pm 3$ & $\mathrm{~N} / \mathrm{A}$ & $\mathrm{N} / \mathrm{A}$ & $11.9 \pm 3.3$ & $9.9 \pm 3.0$ & $10.6 \pm 3.4$ & $10.6 \pm 3.2$ & $N / A$ & N/A \\
\hline SIRS Criteria, No & . /Total no. (\%) & & & & & & & & & \\
\hline $\begin{array}{l}\text { Temperature } \\
\leq 36^{\circ} \mathrm{C} \text { or } \geq 38 \\
{ }^{\circ} \mathrm{C}\end{array}$ & N/A & $\mathrm{N} / \mathrm{A}$ & $\mathrm{N} / \mathrm{A}$ & $\mathrm{N} / \mathrm{A}$ & N/A & N/A & N/A & N/A & $\mathrm{N} / \mathrm{A}$ & N/A \\
\hline $\begin{array}{l}\text { Temperature }{ }^{\circ} \\
\mathrm{C}\end{array}$ & $\mathrm{N} / \mathrm{A}$ & $\mathrm{N} / \mathrm{A}$ & $\mathrm{N} / \mathrm{A}$ & $\mathrm{N} / \mathrm{A}$ & N/A & $\mathrm{N} / \mathrm{A}$ & $37.9 \pm 1.5$ & $38.0 \pm 1.4$ & $38.0 \pm 2$ & $37.9 \pm 2.2$ \\
\hline $\begin{array}{l}\text { Heart rate } \\
\text { Mean } \pm \text { SD or }> \\
90 \text { beats } / \text { min }\end{array}$ & N/A & $\mathrm{N} / \mathrm{A}$ & $96 \pm 21.6$ & $95 \pm 20.9$ & N/A & N/A & $119 \pm 26$ & $118 \pm 25$ & $118 \pm 21$ & $118 \pm 21$ \\
\hline $\begin{array}{l}\text { Mean arterial } \\
\text { pressure - } \\
\mathrm{mm} \mathrm{Hg}\end{array}$ & N/A & $\mathrm{N} / \mathrm{A}$ & $72.5 \pm 8.2$ & $72.2 \pm 8.3$ & $\mathrm{~N} / \mathrm{A}$ & $\mathrm{N} / \mathrm{A}$ & N/A & N/A & $54 \pm 10$ & $55 \pm 10$ \\
\hline $\begin{array}{l}\text { Systolic Blood } \\
\text { Pressure - mm } \\
\mathrm{Hg}\end{array}$ & N/A & $\mathrm{N} / \mathrm{A}$ & $\mathrm{N} / \mathrm{A}$ & $\mathrm{N} / \mathrm{A}$ & $\mathrm{N} / \mathrm{A}$ & N/A & $94 \pm 23$ & $95 \pm 27$ & N/A & N/A \\
\hline $\begin{array}{l}\text { Central venous } \\
\text { pressure - } \\
\mathrm{mm} \mathrm{Hg}\end{array}$ & N/A & $\mathrm{N} / \mathrm{A}$ & $12.0 \pm 5.2$ & $12.1 \pm 5.3$ & $\mathrm{~N} / \mathrm{A}$ & $\mathrm{N} / \mathrm{A}$ & N/A & $\mathrm{N} / \mathrm{A}$ & $\mathrm{N} / \mathrm{A}$ & N/A \\
\hline $\begin{array}{l}\text { Lowest mean } \\
\text { arterial } \\
\text { pressure - } \\
\mathrm{mm} \mathrm{Hg}\end{array}$ & N/A & $\mathrm{N} / \mathrm{A}$ & $57.3 \pm 8.5$ & $57.1 \pm 9.1$ & N/A & N/A & N/A & $N / A$ & $\mathrm{~N} / \mathrm{A}$ & $N / A$ \\
\hline $\begin{array}{l}\text { Highest lactate } \\
\text { level — mg/dl }\end{array}$ & N/A & $\mathrm{N} / \mathrm{A}$ & $34.2 \pm 29.1$ & $34.5 \pm 28.2$ & $\mathrm{~N} / \mathrm{A}$ & N/A & $3.9 \pm 3.6$ & $4.1 \pm 4.1$ & $4.6 \pm 4.4$ & $4.3 \pm 4.3$ \\
\hline igh & & & & & & & & & & \\
\hline
\end{tabular}




\section{Cureus}

\begin{tabular}{|c|c|c|c|c|c|c|c|c|c|c|}
\hline $\begin{array}{l}\text { bilirubin level } \\
\text { — mg/dl }\end{array}$ & $N / A$ & $\mathrm{~N} / \mathrm{A}$ & $1.7 \pm 2.4$ & $1.7 \pm 2.4$ & $N / A$ & $\mathrm{~N} / \mathrm{A}$ & $\mathrm{N} / \mathrm{A}$ & $\mathrm{N} / \mathrm{A}$ & $\mathrm{N} / \mathrm{A}$ & $N / A$ \\
\hline $\begin{array}{l}\text { Highest } \\
\text { creatinine level } \\
-\mathrm{mg} / \mathrm{dl}\end{array}$ & N/A & N/A & $2.2 \pm 2.0$ & $2.1 \pm 1.7$ & N/A & N/A & $\mathrm{N} / \mathrm{A}$ & $\mathrm{N} / \mathrm{A}$ & N/A & N/A \\
\hline $\begin{array}{l}\text { Lowest } \\
\text { Pao2:Fio2 }\end{array}$ & N/A & N/A & $164.6 \pm 91.3$ & $166.4 \pm 91.9$ & N/A & N/A & $162 \pm 89$ & $154 \pm 73$ & $176 \pm 120$ & $171 \pm 124$ \\
\hline $\begin{array}{l}\text { Highest white- } \\
\text { cell count }- \\
\text { cells } \times 10-9 / \text { liter }\end{array}$ & N/A & N/A & $17.4 \pm 11.4$ & $17.8 \pm 14.7$ & $\mathrm{~N} / \mathrm{A}$ & $\mathrm{N} / \mathrm{A}$ & $\mathrm{N} / \mathrm{A}$ & $\mathrm{N} / \mathrm{A}$ & $\mathrm{N} / \mathrm{A}$ & $\mathrm{N} / \mathrm{A}$ \\
\hline $\begin{array}{l}\text { Tachypnea, } \\
\text { hypocapnia, } \\
\text { Mechanical } \\
\text { vent }\end{array}$ & N/A & N/A & N/A & $\mathrm{N} / \mathrm{A}$ & $\mathrm{N} / \mathrm{A}$ & $\mathrm{N} / \mathrm{A}$ & N/A & N/A & N/A & N/A \\
\hline $\begin{array}{l}\text { Leukocytosis, } \\
\text { leukopenia, left } \\
\text { shift }\end{array}$ & N/A & N/A & $\mathrm{N} / \mathrm{A}$ & $\mathrm{N} / \mathrm{A}$ & $N / A$ & $\mathrm{~N} / \mathrm{A}$ & $14.9 \pm 9.8$ & $14.7 \pm 9.8$ & $13.1 \pm 10.1$ & $13.0 \pm 8.4$ \\
\hline $\begin{array}{l}\text { Patients with } \\
\text { comorbidities, } \\
\text { no. }(\%)\end{array}$ & N/A & N/A & $\mathrm{N} / \mathrm{A}$ & $\mathrm{N} / \mathrm{A}$ & 54 (93.1) & 49 (81.7) & $\mathrm{N} / \mathrm{A}$ & $\mathrm{N} / \mathrm{A}$ & N/A & $\mathrm{N} / \mathrm{A}$ \\
\hline Hypertension & $N / A$ & $\mathrm{~N} / \mathrm{A}$ & $\mathrm{N} / \mathrm{A}$ & $\mathrm{N} / \mathrm{A}$ & $25(43.1)$ & $26(43.3)$ & $89(35)$ & $98(40)$ & $44(29)$ & $40(27)$ \\
\hline COPD & $\mathrm{N} / \mathrm{A}$ & $\mathrm{N} / \mathrm{A}$ & $\mathrm{N} / \mathrm{A}$ & N/A & $2(3.4)$ & $4(6.7)$ & $27(11)$ & $29(12)$ & $17(11)$ & $24(16)$ \\
\hline CAD & $N / A$ & $\mathrm{~N} / \mathrm{A}$ & $\mathrm{N} / \mathrm{A}$ & $\mathrm{N} / \mathrm{A}$ & $7(12.1)$ & $8(13.3)$ & $37(15)$ & $47(19)$ & $20(13)$ & $11(7)$ \\
\hline DM & $\mathrm{N} / \mathrm{A}$ & $\mathrm{N} / \mathrm{A}$ & $\mathrm{N} / \mathrm{A}$ & $\mathrm{N} / \mathrm{A}$ & $14(24.1)$ & $12(20.0)$ & $51(20)$ & $56(23)$ & $20(13)$ & $17(11)$ \\
\hline CKD & $N / A$ & $\mathrm{~N} / \mathrm{A}$ & N/A & $\mathrm{N} / \mathrm{A}$ & $2(3.4)$ & $1(1.7)$ & $22(9)$ & $21(9)$ & & \\
\hline Malignancy & $\mathrm{N} / \mathrm{A}$ & N/A & $\mathrm{N} / \mathrm{A}$ & N/A & $9(15.5)$ & $13(21.7)$ & $47(19)$ & $37(15)$ & $23(15)$ & $18(12)$ \\
\hline $\begin{array}{l}\text { Community } \\
\text { Acquired } \\
\text { Infection }\end{array}$ & $468(77.7)$ & 459 (75.5) & $\mathrm{N} / \mathrm{A}$ & N/A & N/A & N/A & $\mathrm{N} / \mathrm{A}$ & $\mathrm{N} / \mathrm{A}$ & $94(63)$ & $93(62)$ \\
\hline $\begin{array}{l}\text { Nosocomial, } \\
\text { ICU }\end{array}$ & $N / A$ & $N / A$ & N/A & N/A & N/A & N/A & N/A & N/A & N/A & N/A \\
\hline $\begin{array}{l}\text { Nosocomial, } \\
\text { Ward }\end{array}$ & $N / A$ & $N / A$ & N/A & N/A & N/A & $\mathrm{N} / \mathrm{A}$ & $N / A$ & N/A & N/A & $\mathrm{N} / \mathrm{A}$ \\
\hline Nosocomial & $N / A$ & $N / A$ & N/A & N/A & $\mathrm{N} / \mathrm{A}$ & $\mathrm{N} / \mathrm{A}$ & $\mathrm{N} / \mathrm{A}$ & N/A & $30(20)$ & $34(23)$ \\
\hline Site of Infection $n$ & o. $(\%)$ & & & & & & & & & \\
\hline Unknown & $11(1.8)$ & $18(2.9)$ & $145(7.9)$ & $136(7.3)$ & $7(12.1)$ & $4(6.1)$ & $\mathrm{N} / \mathrm{A}$ & $\mathrm{N} / \mathrm{A}$ & $2(1)$ & 0 \\
\hline Lung & $373(60.7)$ & $363(58)$ & $623(33.8)$ & $677(36.5)$ & $22(37.9)$ & $22(36.7)$ & $\mathrm{N} / \mathrm{A}$ & $\mathrm{N} / \mathrm{A}$ & $61(41)$ & $70(47)$ \\
\hline Abdomen & $74(12.1)$ & 68 (10.9) & 477 (25.9) & $467(25.2)$ & $21(36.2)$ & $34(56.7)$ & $\mathrm{N} / \mathrm{A}$ & N/A & $26(17)$ & $23(15)$ \\
\hline Urinary Tract & $102(16.6)$ & $118(18.8)$ & $146(146(7.9)$ & $133(7.2)$ & $10(17.2)$ & $7(11.7)$ & $\mathrm{N} / \mathrm{A}$ & $\mathrm{N} / \mathrm{A}$ & $7(5)$ & $7(5)$ \\
\hline $\begin{array}{l}\text { Skin and soft } \\
\text { tissues }\end{array}$ & $N / A$ & $N / A$ & $137(7.4)$ & $116(6.3)$ & $2(3.4)$ & $1(1.7)$ & N/A & N/A & $8(5)$ & $12(8)$ \\
\hline Bacteremia & $N / A$ & $\mathrm{~N} / \mathrm{A}$ & $\mathrm{N} / \mathrm{A}$ & $\mathrm{N} / \mathrm{A}$ & $18(31.0)$ & $13(21.7)$ & $\mathrm{N} / \mathrm{A}$ & $\mathrm{N} / \mathrm{A}$ & $39(26)$ & $31(21)$ \\
\hline Surgical wound & $N / A$ & N/A & $\mathrm{N} / \mathrm{A}$ & $\mathrm{N} / \mathrm{A}$ & $\mathrm{N} / \mathrm{A}$ & $\mathrm{N} / \mathrm{A}$ & $\mathrm{N} / \mathrm{A}$ & $\mathrm{N} / \mathrm{A}$ & $\mathrm{N} / \mathrm{A}$ & $N / A$ \\
\hline $\begin{array}{l}\text { Positive blood } \\
\text { culture no. (\%) }\end{array}$ & $225(36.6)$ & $229(36.6)$ & 316 (1.1) & $325(17.5)$ & $42(72.4)$ & 44 (73.3) & $\mathrm{N} / \mathrm{A}$ & $\mathrm{N} / \mathrm{A}$ & $39(26)$ & $31(21)$ \\
\hline $\begin{array}{l}\text { Documented } \\
\text { pathogen no. } \\
(\%)\end{array}$ & $450(73.3)$ & $441(70.4)$ & $\mathrm{N} / \mathrm{A}$ & $\mathrm{N} / \mathrm{A}$ & N/A & N/A & N/A & N/A & N/A & N/A \\
\hline $\begin{array}{l}\text { Gram-positive } \\
\text { bacteria no. (\%) }\end{array}$ & $235(38.3)$ & $228(36.4)$ & N/A & N/A & $4(6.9)$ & $4(6.7)$ & N/A & N/A & $46(31)$ & 37 (25) \\
\hline
\end{tabular}




\section{Cureus}

\begin{tabular}{|c|c|c|c|c|c|c|c|c|c|c|}
\hline $\begin{array}{l}\text { Gram-negative } \\
\text { bacteria no. (\%) }\end{array}$ & $261(42.5)$ & $264(42.2)$ & N/A & N/A & $26(44.9)$ & $31(51.7)$ & $\mathrm{N} / \mathrm{A}$ & N/A & $37(25)$ & $45(30)$ \\
\hline $\begin{array}{l}\text { Adequate } \\
\text { antimicrobial } \\
\text { therapy no. (\%) }\end{array}$ & 595 (96.9) & $595(96.2)$ & 1817 (98.3) & 1821 (98.1) & $48(82.8)$ & $47(78.3)$ & $\mathrm{N} / \mathrm{A}$ & N/A & 137 (91) & 141 (95) \\
\hline \multicolumn{11}{|c|}{ Vasopressor administration } \\
\hline \multicolumn{11}{|l|}{ Epinephrine } \\
\hline No. of patients & 53 & 58 & 134 & 113 & N/A & N/A & $35(14)$ & $22(9)$ & 41 & 31 \\
\hline $\begin{array}{l}\text { Dose - } \\
\mu \mathrm{g} / \mathrm{kg} / \mathrm{min}\end{array}$ & $2.31 \pm 6.62$ & $1.74 \pm 2.41$ & $\mathrm{~N} / \mathrm{A}$ & N/A & N/A & $\mathrm{N} / \mathrm{A}$ & $0.6 \pm 1.2$ & $0.9 \pm 2.6$ & $0.8 \pm 0.7$ & $1 \pm 0.9$ \\
\hline \multicolumn{11}{|l|}{ Norepinephrine } \\
\hline No. of patients & 534 & 554 & 1823 & 1821 & $N / A$ & $\mathrm{~N} / \mathrm{A}$ & 224(89) & $231(93)$ & 46 & 48 \\
\hline $\begin{array}{l}\text { Dose - } \\
\mu \mathrm{g} / \mathrm{kg} / \mathrm{min}\end{array}$ & $1.02 \pm 1.61$ & $1.14 \pm 1.66$ & $\mathrm{~N} / \mathrm{A}$ & N/A & $1.7 \pm 2.1$ & $1.2 \pm 1.4$ & $0.5 \pm 0.6$ & $0.4 \pm 0.5$ & $1.1 \pm 1.1$ & $1.0 \pm 1.1$ \\
\hline \multicolumn{11}{|l|}{ Glucocorticoids } \\
\hline $\begin{array}{l}\text { IV No./Total } \\
\text { No. (\%) }\end{array}$ & N/A & N/A & N/A & N/A & $\mathrm{N} / \mathrm{A}$ & N/A & N/A & N/A & N/A & $N / A$ \\
\hline $\begin{array}{l}\text { Hydrocortisone } \\
\text { equivalent, } \\
\text { (range), mg }\end{array}$ & $\mathrm{N} / \mathrm{A}$ & N/A & N/A & N/A & N/A & N/A & $N / A$ & N/A & $\mathrm{N} / \mathrm{A}$ & $\mathrm{N} / \mathrm{A}$ \\
\hline \multicolumn{11}{|l|}{ Etomidate } \\
\hline $\begin{array}{l}\text { No. / Total no. } \\
\text { (\%) }\end{array}$ & $\mathrm{N} / \mathrm{A}$ & $\mathrm{N} / \mathrm{A}$ & $N / A$ & N/A & N/A & $\mathrm{N} / \mathrm{A}$ & 22/251(8.6) & 20/248(8.1) & N/A & $\mathrm{N} / \mathrm{A}$ \\
\hline Mean (SD), mg & $N / A$ & $\mathrm{~N} / \mathrm{A}$ & $\mathrm{N} / \mathrm{A}$ & N/A & $\mathrm{N} / \mathrm{A}$ & $\mathrm{N} / \mathrm{A}$ & $N / A$ & $\mathrm{~N} / \mathrm{A}$ & $\mathrm{N} / \mathrm{A}$ & $\mathrm{N} / \mathrm{A}$ \\
\hline $\begin{array}{l}\text { Mechanical } \\
\text { ventilation no. } \\
(\%)\end{array}$ & $567(92.3)$ & $569(91.3)$ & 1845 (99.8) & 1855 (99.9) & $52(89.7)$ & $51(85.0)$ & 228(91) & 212(86) & $87(58)$ & $75(50.3)$ \\
\hline $\begin{array}{l}\text { Renal- } \\
\text { replacement } \\
\text { therapy no. (\%) }\end{array}$ & $161(27)$ & $168(28.1)$ & $228(12.3)$ & $242(13.0)$ & $24(41.4)$ & $18(30.0)$ & $\mathrm{N} / \mathrm{A}$ & N/A & N/A & $\mathrm{N} / \mathrm{A}$ \\
\hline $\begin{array}{l}\text { Organ failure } \mathrm{n} \text {. } \\
(\%)\end{array}$ & $\mathrm{N} / \mathrm{A}$ & N/A & $\mathrm{N} / \mathrm{A}$ & N/A & $10(17.2)$ & $6(10.0)$ & $\mathrm{N} / \mathrm{A}$ & N/A & N/A & $\mathrm{N} / \mathrm{A}$ \\
\hline Respiratory & $N / A$ & $\mathrm{~N} / \mathrm{A}$ & $\mathrm{N} / \mathrm{A}$ & $\mathrm{N} / \mathrm{A}$ & $7(12.1)$ & $4(6.7)$ & $N / A$ & $\mathrm{~N} / \mathrm{A}$ & $\mathrm{N} / \mathrm{A}$ & $\mathrm{N} / \mathrm{A}$ \\
\hline Liver & $N / A$ & $\mathrm{~N} / \mathrm{A}$ & N/A & $\mathrm{N} / \mathrm{A}$ & $1(1.7)$ & $1(1.7)$ & $N / A$ & $\mathrm{~N} / \mathrm{A}$ & $\mathrm{N} / \mathrm{A}$ & $\mathrm{N} / \mathrm{A}$ \\
\hline Renal & N/A & N/A & N/A & N/A & $3(5.2)$ & $1(1.7)$ & N/A & N/A & $\mathrm{N} / \mathrm{A}$ & N/A \\
\hline Coagulation & $N / A$ & $N / A$ & $\mathrm{~N} / \mathrm{A}$ & $\mathrm{N} / \mathrm{A}$ & $3(5.2)$ & $1(1.7)$ & $N / A$ & N/A & $\mathrm{N} / \mathrm{A}$ & $\mathrm{N} / \mathrm{A}$ \\
\hline Microcirculatory & $N / A$ & $\mathrm{~N} / \mathrm{A}$ & $\mathrm{N} / \mathrm{A}$ & N/A & $\mathrm{N} / \mathrm{A}$ & $\mathrm{N} / \mathrm{A}$ & $N / A$ & $\mathrm{~N} / \mathrm{A}$ & $\mathrm{N} / \mathrm{A}$ & $N / A$ \\
\hline $\begin{array}{l}\text { Central nervous } \\
\text { system }\end{array}$ & $\mathrm{N} / \mathrm{A}$ & N/A & $\mathrm{N} / \mathrm{A}$ & N/A & N/A & N/A & $N / A$ & N/A & $\mathrm{N} / \mathrm{A}$ & $\mathrm{N} / \mathrm{A}$ \\
\hline
\end{tabular}

\section{TABLE 3: Baseline Characteristics of Individuals Trials}

no. $=$ Number, $\mathrm{SD}=$ Standard Deviation; SAPS $=$ Simplified Acute Physiology Score; SOFA $=$ Sequential Organ Failure Assessment; APACHE $=$ Acute Physiology and Chronic Health Evaluation; SIRS = Systemic Inflammatory Response Syndrome; Pao2:Fio2 = the ratio of the partial pressure of arterial oxygen to the fraction of inspired oxygen; $C O P D=$ Chronic Obstructive Pulmonary Disease; $C A D=$ Coronary Artery Disease; $D M=$ Diabetes Mellitus: $\mathrm{CKD}=$ Chronic Kidney Disease; ICU = Intensive Care Unit; IV = Intravenous; N/A = Data not available

\section{Data synthesis and analysis}

Statistical Method 


\section{Cureus}

We used a random effects model for our statistical analysis in RevMan Version 5.3 Copenhagen. We used the Mantel-Haenszel method for the statistical analysis of dichotomous data to calculate the risks ratio and inverse variance for the continuous data to estimate the mean difference. We reported our results using the effect estimate with $95 \%$ confidence interval. A two-sided p-value of $\leqslant 0.05$ was considered statistically significant.

\section{Heterogeneity}

We used I2 and Chi2 statistics to estimate the heterogeneity with RevMan Version 5.3 Copenhagen. Variability between studies (inter-study) compared to variability within studies (intra-study) was assessed with the I2 statistic; I2 >50\% indicates substantial heterogeneity as mentioned in the Cochrane Handbook for Systematic Reviews for Interventions, Version 5.1.0, Part 2: General Methods for Cochrane Reviews [19]. We performed a sensitivity analysis for substantial heterogeneity.

Study Selection

We identified 244 citations for RCTs. Two reviewers W.J.S. and P.I. reviewed the abstracts of each study and selected 25 articles and reviewed their full papers. They excluded 20 papers and selected five articles for qualitative and quantitative analysis comparing hydrocortisone to the placebo in patients with septic shock. Figure 1 shows the PRISMA study flow diagram and Table 4 summarizes the excluded studies failing to meet the inclusion criteria.

\begin{tabular}{|c|c|c|c|c|c|c|}
\hline Name & Random Sequence & $\begin{array}{l}\text { Allocation } \\
\text { Concealment }\end{array}$ & $\begin{array}{l}\text { Blinding of } \\
\text { Participants } \\
\text { and } \\
\text { Personnel }\end{array}$ & $\begin{array}{l}\text { Blinding of } \\
\text { Outcome } \\
\text { Assessment }\end{array}$ & Incomplete Outcome Data & $\begin{array}{l}\text { Selective } \\
\text { Reporting }\end{array}$ \\
\hline $\begin{array}{l}2018 \\
\text { APROCCHSS } \\
\text { trial }[14]\end{array}$ & $\begin{array}{l}\text { Yes Via Centralized } \\
\text { Randomization Web site, } \\
\text { stratified using permutation } \\
\text { blocks Low Risk }\end{array}$ & Yes Low Risk & Yes Low Risk & $\begin{array}{l}\text { Yes Low } \\
\text { Risk }\end{array}$ & No Low Risk & $\begin{array}{l}\text { No Low } \\
\text { Risk }\end{array}$ \\
\hline $\begin{array}{l}2018 \\
\text { ADRENAL } \\
\text { trial [13] }\end{array}$ & $\begin{array}{l}\text { Yes Password-protected, } \\
\text { encrypted, Web-based Interface } \\
\text { Low Risk }\end{array}$ & Yes Low Risk & Yes Low Risk & $\begin{array}{l}\text { Yes Low } \\
\text { Risk }\end{array}$ & No Low Risk & $\begin{array}{l}\text { No Low } \\
\text { Risk }\end{array}$ \\
\hline $\begin{array}{l}2017 \text { Qing- } \\
\text { quan Lv et } \\
\text { al. [11] }\end{array}$ & $\begin{array}{l}\text { Yes Computer-generated } \\
\text { random numbers Low Risk }\end{array}$ & $\begin{array}{l}\text { Not Reported } \\
\text { Unclear }\end{array}$ & Yes Low Risk & $\begin{array}{l}\text { Not Reported } \\
\text { Unclear }\end{array}$ & No Low Risk & $\begin{array}{l}\text { No Low } \\
\text { Risk }\end{array}$ \\
\hline $\begin{array}{l}2008 \\
\text { CORTICUS } \\
\text { trial [17] }\end{array}$ & $\begin{array}{l}\text { Yes Computerized random- } \\
\text { number generator Low Risk }\end{array}$ & Yes Low Risk & Yes Low Risk & $\begin{array}{l}\text { Yes Low } \\
\text { Risk }\end{array}$ & Yes High Risk & $\begin{array}{l}\text { No Low } \\
\text { Risk }\end{array}$ \\
\hline $\begin{array}{l}2002 \text { Annane } \\
\text { et al. [9] }\end{array}$ & $\begin{array}{l}\text { Yes Computer-generated } \\
\text { random number Low Risk }\end{array}$ & Yes Low Risk & Yes Low Risk & $\begin{array}{l}\text { Yes Low } \\
\text { Risk }\end{array}$ & $\begin{array}{l}\text { Yes One person withdrew consent } \\
\text { after getting assigned treatment was } \\
\text { excluded from analysis High Risk }\end{array}$ & $\begin{array}{l}\text { No Low } \\
\text { Risk }\end{array}$ \\
\hline
\end{tabular}

TABLE 4: Cochrane Risk of Bias for Quality Assessment

Qualitative Analysis

We included five RCTs with 5,838 patients in our analysis. 2,914 patients were randomized to the hydrocortisone arm vs. 2,924 to the placebo arm. Two studies used Fludrocortisone in addition to hydrocortisone in the steroid arm $[9,14]$. (Table 5) 


\section{Cureus}

\begin{tabular}{|c|c|c|c|c|c|c|c|c|}
\hline $\begin{array}{l}\text { Total } \\
\text { Studies }\end{array}$ & $\begin{array}{l}\text { Studies } \\
\text { Included }\end{array}$ & Studies Excluded & & & & & & \\
\hline 244 & 5 & 239 & & & & & & \\
\hline $\begin{array}{l}\text { Exclusion } \\
\text { Criteria }\end{array}$ & $\begin{array}{l}\text { Non- } \\
\text { Randomized } \\
\text { Studies }\end{array}$ & $\begin{array}{l}\text { Hydrocortisone or } \\
\text { Fludrocortisone }\end{array}$ & $\begin{array}{l}\text { studies } \\
\text { less than } \\
100\end{array}$ & $\begin{array}{l}\text { of steroids or } \\
\text { Septic Shock }\end{array}$ & $\begin{array}{l}\text { Non-Englisn } \\
\text { Language } \\
\text { Studies }\end{array}$ & $\begin{array}{l}\text { Primary Outcome i.e. } \\
\text { 28-day mortality }\end{array}$ & $\begin{array}{l}\text { were Study } \\
\text { Designs/Protocols }\end{array}$ & $\begin{array}{l}\text { Age } \\
<18 \\
\text { years }\end{array}$ \\
\hline n. & 8 & 8 & 4 & 199 & 1 & 4 & 4 & 11 \\
\hline
\end{tabular}

TABLE 5: Summary of Studies Excluded

\section{Results \\ Primary endpoints}

See Table 1 .

Mortality at 28 Days

There was a total of 1,688 deaths with a significantly reduced number of deaths in the hydrocortisone and fludrocortisone arm as compared to the placebo arm. There were 808 deaths in the hydrocortisone arm vs. 880 in the placebo arm, risk ratio $(R R)=0.92$, confidence interval $(\mathrm{CI})=0.85-0.99, \mathrm{p}=0.04, \mathrm{I} 2=0 \%$, suggesting the mortality benefit at 28 days with hydrocortisone and fludrocortisone in septic shock patients. The sub-group analysis of the early administration of hydrocortisone, i.e., within eight hours of randomization showed no difference between the two groups, 105 in hydrocortisone group vs. 110 in the placebo arm, $\mathrm{RR}=0.98, \mathrm{CI}=0.73-1.32, \mathrm{p}=0.90, \mathrm{I} 2=38 \%$. The sub-group analysis of the late administration of hydrocortisone, i.e., within 24-72 hours of randomization showed a non-significant trend towards decreased mortality in the hydrocortisone arm, 703 vs. 770 in the placebo arm, RR $=0.92, \mathrm{CI}=0.83-1.01, \mathrm{p}=$ $0.09, \mathrm{I} 2=16 \%$ (Figure 2).

\section{Secondary endpoints}

See Table 1.

\section{Mortality in the ICU}

There was a total of 856 deaths in the ICU with no difference in the number of deaths between the two groups, the hydrocortisone arm (409) vs. the placebo $\operatorname{arm}(447), \mathrm{RR}=0.93, \mathrm{CI}=0.81-1.08, \mathrm{p}=0.35, \mathrm{I} 2=$ $52 \%$ (Figure 3). There was substantial heterogeneity between the two groups. On running the sensitivity analysis without the results of the Corticosteroid Therapy of Septic Shock (CORTICUS) trial, the results became statistically significant favoring hydrocortisone and fludrocortisone with I2 reducing to $0 \%$. Hydrocortisone $\operatorname{arm}=307$ vs. placebo $\operatorname{arm}=358 \mathrm{RR}=0.87, \mathrm{CI}=0.78-0.97, \mathrm{p}=0.01, \mathrm{I} 2=0 \%$.

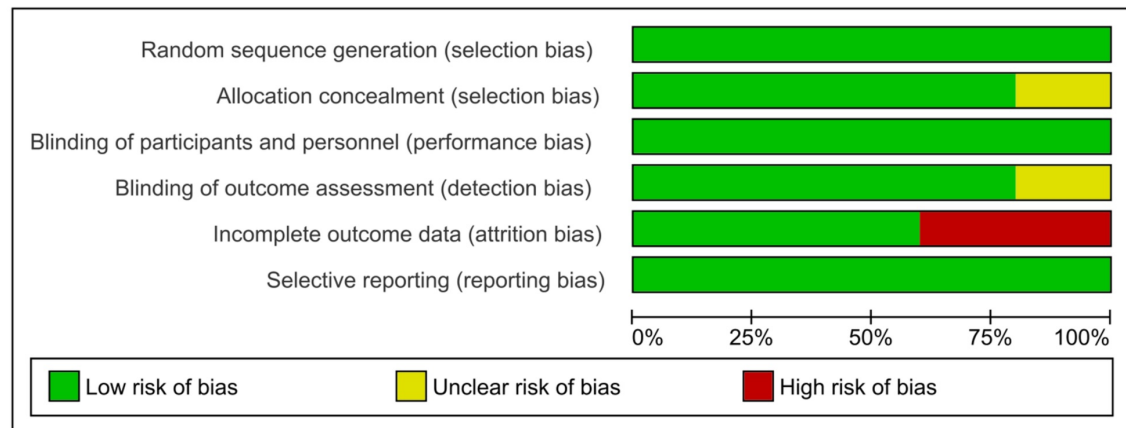

FIGURE 3: Risk of Bias Graph

Mortality in the Hospital

There were a total of 974 deaths during the hospital stay with no difference in either arm, 468 in the 


\section{Cureus}

hydrocortisone arm vs. 506 in the placebo $\mathrm{arm}, \mathrm{RR}=0.95, \mathrm{CI}=0.84-1.08, \mathrm{p}=0.41, \mathrm{I} 2=39 \%$ (Figure 4 ).

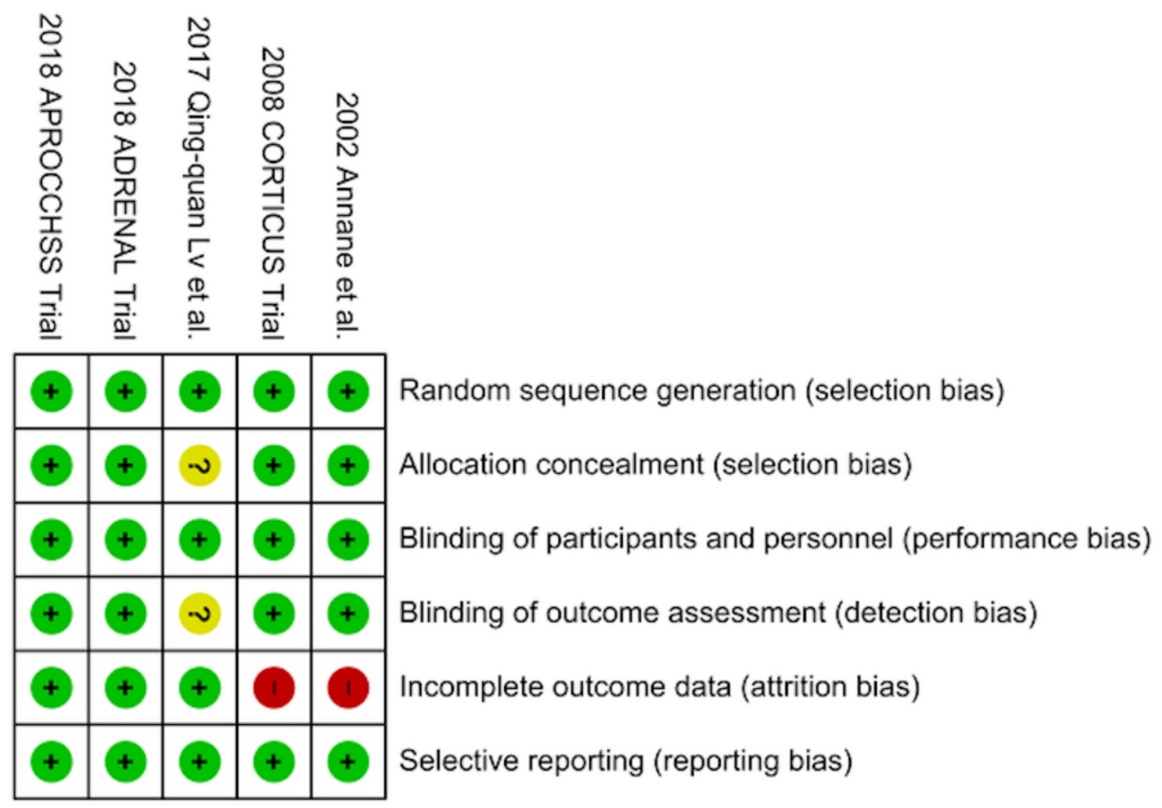

FIGURE 4: Showing Risk of Bias Summary

Mortality at 90 Days

Two trials reported 90-day mortality. There was a non-significant trend towards decreased mortality at 90 days in the hydrocortisone and fludrocortisone group, $775 \mathrm{vs} .834$ in the placebo arm, RR $=0.93, \mathrm{CI}=0.84$ $1.02, \mathrm{p}=0.13, \mathrm{I} 2=37 \%$ (Figure 5).

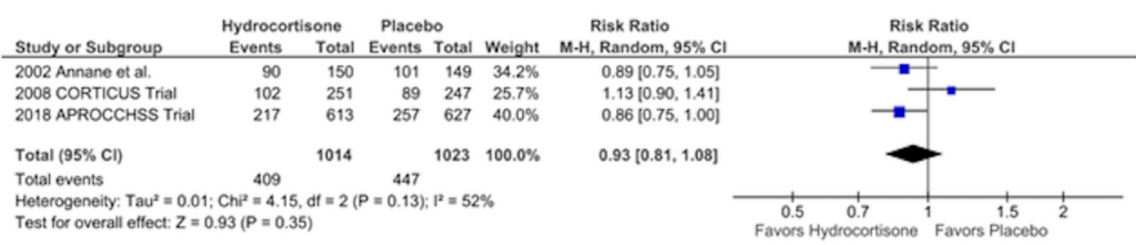

FIGURE 5: Forest Plot Showing Mortality in the Intensive Care Unit

Mortality at One Year

Two studies reported one-year mortality. There was no difference in mortality between the two groups at one year, 239 deaths in each $\mathrm{arm}, \mathrm{RR}=0.97, \mathrm{CI}=0.84-1.12, \mathrm{p}=0.67, \mathrm{I} 2=46 \%$ (Figure 6 ).

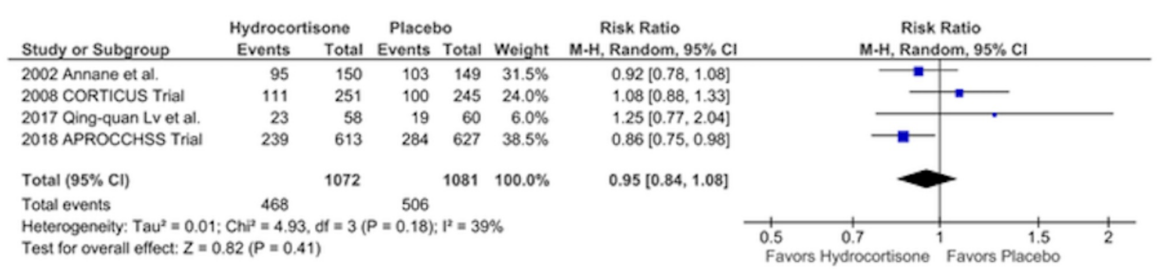

FIGURE 6: Forest Plot Showing Mortality in the Hospital

Reversal of Shock

Two studies reported the shock reversal outcome. A total of 238 patients in the hydrocortisone group had shock reversal as compared to 226 in the placebo arm. There was no statistical difference between the two 


\section{Cureus}

groups, $\mathrm{OR}=1.17, \mathrm{CI}=0.74-1.86, \mathrm{p}=0.50, \mathrm{I} 2=24 \%$ (Figure 7 ).

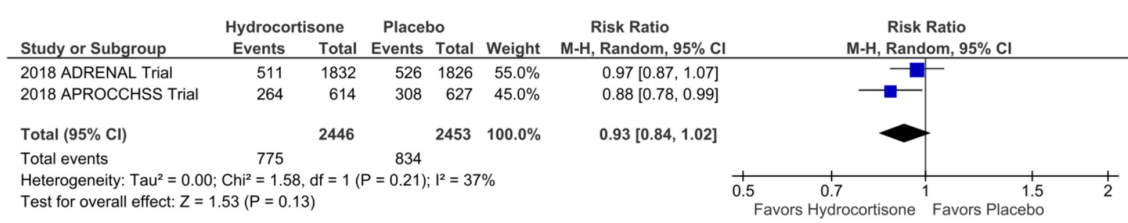

FIGURE 7: Forest Plot Showing Mortality at 90 Days

ICULOS

Only two studies reported the ICU LOS. There was no difference in the LOS in the ICU between the two groups, point estimate $=0.89$ days, $\mathrm{CI}=-2.56$ to $4.33, \mathrm{p}=0.61, \mathrm{I}^{2}=0 \%$ (Figure 8 ).

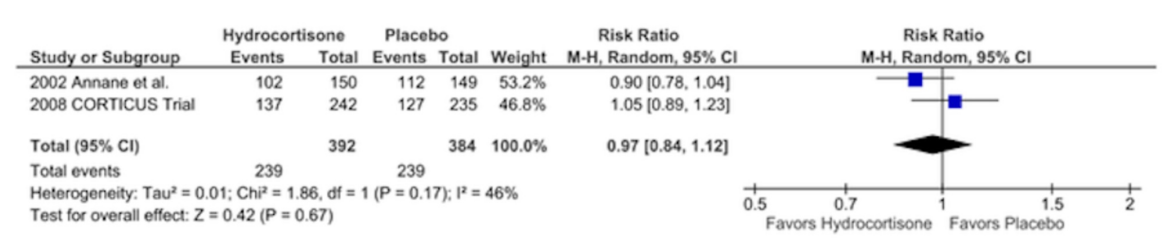

FIGURE 8: Forest Plot Showing Mortality at One Year

Hospital LOS

Two studies reported the LOS in the hospital. No statistical difference was observed in the two arms, point estimate $=1.58$ days, $\mathrm{CI}=-4.23$ to $7.38, \mathrm{p}=0.59, \mathrm{I}^{2}=0 \%$ (Figure 9 ).

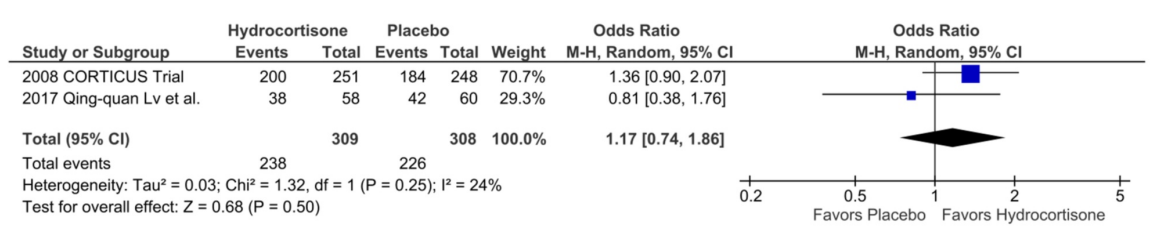

FIGURE 9: Forest Plot Showing Reversal of Shock

Incidence of Limb and/or Cerebral Ischemia

Two studies reported the incidence of either limb and/or cerebral ischemia. There was no difference in the two groups, four in the hydrocortisone arm as compared to three in the placebo arm, RR $=1.32, \mathrm{CI}=0.30$ $5.9, \mathrm{p}=0.72, \mathrm{I}^{2}=0 \%$ (Figure 10 ).

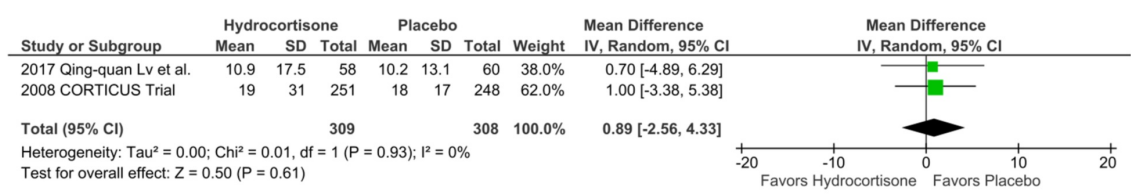

FIGURE 10: Forest Plot Showing the Length of Stay in the Intensive Care Unit

Incidence of Superinfection

Three studies reported the incidence of superinfection in the two treatment arms. Hydrocortisone was associated with a significantly higher number of superinfections as compared to placebo, $436 \mathrm{vs.} 385$, RR = 


\section{Cureus}

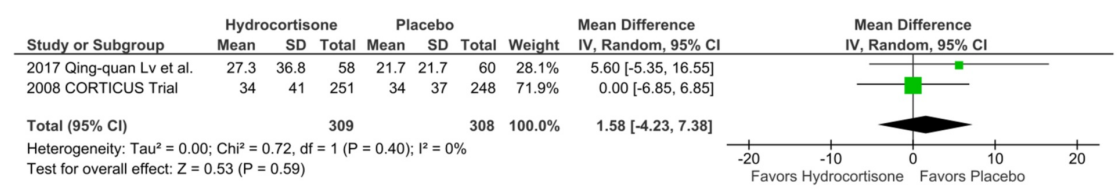

FIGURE 11: Forest Plot Showing the Length of Stay in the Hospital

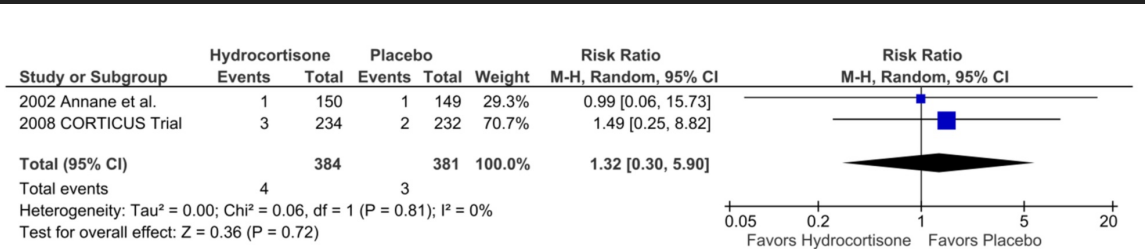

FIGURE 12: Forest Plot Showing the Incidence of Limb and/or Cerebral Ischemia

\begin{tabular}{|c|c|c|c|c|c|c|c|c|c|}
\hline Study or Subgroup & \multicolumn{2}{|c|}{ Hydrocortisone } & \multicolumn{2}{|c|}{ Placebo } & Weight & $\begin{array}{c}\text { Risk Ratio } \\
\text { M-H, Random, } 95 \% \mathrm{Cl}\end{array}$ & \multicolumn{2}{|c|}{$\begin{array}{l}\text { Risk Ratio } \\
\text { M-H, Random, } 95 \% \mathrm{Cl}\end{array}$} & \\
\hline 2002 Annane et al. & 32 & 150 & 33 & 149 & $5.5 \%$ & $0.96[0.63,1.48]$ & & & \\
\hline 2008 CORTICUS Trial & 78 & 234 & 61 & 232 & $12.9 \%$ & $1.27[0.96,1.68]$ & & & \\
\hline 2018 APROCCHSS Trial & 326 & 614 & 291 & 626 & $81.5 \%$ & $1.14[1.02,1.28]$ & & & \\
\hline Total $(95 \% \mathrm{Cl})$ & & 998 & & 1007 & $100.0 \%$ & $1.15[1.04,1.27]$ & & & \\
\hline \multirow{2}{*}{\multicolumn{7}{|c|}{$\begin{array}{l}\text { Total events } \\
\text { Heterogeneity: } \text { Tau }^{2}=0.00 ; \mathrm{Chi}^{2}=1.12, \mathrm{df}=2(\mathrm{P}=0.57) ; \mathrm{l}^{2}=0 \% \\
\text { Test for overall effect: } Z=2.65(P=0.008)\end{array}$}} & & & \\
\hline & & & & & & & 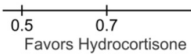 & ${ }^{1}$ Favors Placebo $\begin{array}{r}1.5 \\
\text {. }\end{array}$ & 2 \\
\hline
\end{tabular}

FIGURE 13: Forest Plot Showing the Incidence of Superinfections

\section{Discussion}

The use of steroids in septic shock patients remains controversial due to the inconsistent results of previous trials and meta-analyses. Some authors believe that the significant variation in results could be due to dosing, duration of administration, and timing of initiation of steroids [20-21]. In pre-1997 trials, steroids were used in higher doses for a shorter duration. Subsequent studies were done after 1997 used steroids in physiological doses but for a longer duration, i.e., seven days. In the landmark study by Annane et al., steroids were started within three to eight hours after the diagnosis of septic shock, which led to a significant reduction in mortality [9]. However, in the CORTICUS trial, steroids were started within 12 hours of diagnosis, and no mortality benefit was observed [17]. Nonetheless, both of these trials showed an early reversal of shock in the steroid group. Due to the controversies surrounding the timing of steroid use noted in previous studies, Qing-Quan Lv et al. initiated steroids at the same time when vasopressors were started and found that reversal of shock was similar in both groups and there was no mortality difference either [11]. In the two recent landmark trials, ADRENAL (Adjunctive Corticosteroid Treatment in Critically Ill Patients with Septic Shock) and APROCCHSS (Activated Protein C and Corticosteroids for Human Septic Shock), steroids were started four and six hours after the initiation of vasopressors. Both trials also showed a reduction in pressor requirement with the use of steroids. Another point of debate has been regarding continuous infusion versus intermittent bolus dosing. The HYPRESS (Hydrocortisone for Prevention of Septic Shock) trial, which randomized patients with severe sepsis before developing septic shock, studied the continuous infusion of hydrocortisone with a taper over six days. It also failed to uncover any significant mortality benefit nor did it prevent the development of septic shock [12]. However, the surviving sepsis campaign guidelines recommend only intermittent bolus doses of hydrocortisone and not continuous infusion [2].

Amidst all this confusion, recently, two large multicenter RCTs were published to confirm or refute the findings of previous studies. In the ADRENAL trial, nearly 3,800 patients were randomized and assigned to receive a continuous infusion of either hydrocortisone or placebo. This study was adequately powered to determine a mortality difference [13]. In the APROCCHSS trial, a total of 1,241 patients were randomized to receive either a hydrocortisone - fludrocortisone combination or placebo [14]. The primary outcome in both trials was mortality at 90 days. The ADRENAL trial showed no significant mortality benefit at 90 days; on the other hand, in the APROCCHSS trial, a mortality benefit was noted in the hydrocortisone and fludrocortisone group. Both trials did show an early reversal of shock and rapid cessation of mechanical ventilation, which was similar to the results of earlier studies $[9,17]$. 
A review of these trials provides some additional insights into the subsets of patients who might benefit from the addition of corticosteroid therapy on top of conventional treatment for sepsis. The RCT with lower overall mortality (HYPRESS) had no mortality benefit, likely due to less sick patients (severe sepsis vs. septic shock) [12], and the trials with the highest mortality (French, APROCHSS) likely with the sickest patients, showed a mortality benefit $[9,14]$. This suggests that the addition of steroids may be helpful in patients who are "sicker," and, in this case, unresponsive to conventional therapy of fluids, vasopressors, and antibiotics. Additionally, in the ADRENAL trial, there was a mortality benefit in the sub-group that received steroids after the first six hours, before which the patient, otherwise responsive to conventional goaldirected therapy, would be "selected out," leaving patients who may have an additional benefit from corticosteroid therapy. Similarly, our sub-group analysis of the primary outcome on the basis of time to administration of hydrocortisone from randomization suggested a strong trend towards reduced mortality in the late administration group as compared to the early administration group (within eight hours vs. within 24 to 72 hours). Since the trials in the late administration group may also have patients who received hydrocortisone within eight hours of randomization could have led to a non-significant trend towards decreased mortality. These observations would remain speculative in the absence of a randomized trial looking at these specific outcomes and warrant a randomized clinical trial looking at hydrocortisone use in patients who are unresponsive to early goal-directed therapy. Such a trial would indeed be challenging to design and implement, given logistic and ethical issues.

Our study noted a statistically significant mortality benefit of the primary outcome of mortality at 28 days in the hydrocortisone group as compared to placebo. Also, there was a non-significant reduction in ICU mortality in the hydrocortisone arm, which became statistically significant after sensitivity analysis. This finding also suggests that the use of hydrocortisone incurs a mortality benefit in the ICU setting in addition to 28 days. However, the rates of superinfection were noted to be higher in the hydrocortisone group as compared to the placebo, which is consistent with the results of individual trials and older studies. The strength of our analysis primarily lies in study selection. We included only RCTs, which included patients with a septic shock, which compared hydrocortisone to placebo, and studies with at least a hundred patients in the trial. This is in contrast to earlier meta-analyses, which also included non-RCTs, cohort and retrospective studies, and studies with small population sizes with different steroids, including methylprednisolone, dexamethasone, betamethasone, hydrocortisone, and prednisone [10,20].

The limitations in performing this meta-analysis are: we included trials ranging from 2002 up until now. The management of sepsis and septic shock has evolved since early 2000, and so have the surviving sepsis guidelines [2,22-25], which is evident by the reduction in mortality from $57.8 \%$ in the French study [9] to $23.3 \%$ to $35.8 \%$ in subsequent studies $[11,13-14,17]$. Two out of five trials included fludrocortisone in addition to hydrocortisone. The recommendations from the American College of Critical Care Medicine and the surviving sepsis campaign in 2008 have reported that hydrocortisone has enough mineralocorticoid effects, making the administration of fludrocortisone irrelevant. Thus, we believe that the addition of fludrocortisone could not have provided a significant benefit to influence the results of our study [22,25]. The last limitation was the way steroids were administered in individual RCTs, bolus vs. continuous infusion.

\section{Conclusions}

Our analysis showed a significant reduction in mortality at 28 days and a non-significant trend in ICU mortality and mortality at 90 days in the hydrocortisone group. The rates of superinfection were noted to be significantly higher in the hydrocortisone group. We believe there is still controversy over hydrocortisone, and we don't know which patients, if any, should receive the drug. In the future, large RCTs are required before any new recommendations can be made comparing hydrocortisone to placebo, hydrocortisone plus fludrocortisone to placebo, and hydrocortisone to hydrocortisone plus fludrocortisone. Another potential trial can be designed comparing the time from the development of septic shock to the administration of hydrocortisone.

\section{Additional Information \\ Disclosures}

Human subjects: All authors have confirmed that this study did not involve human participants or tissue. Animal subjects: All authors have confirmed that this study did not involve animal subjects or tissue. Conflicts of interest: In compliance with the ICMJE uniform disclosure form, all authors declare the following: Payment/services info: All authors have declared that no financial support was received from any organization for the submitted work. Financial relationships: All authors have declared that they have no financial relationships at present or within the previous three years with any organizations that might have an interest in the submitted work. Other relationships: All authors have declared that there are no other relationships or activities that could appear to have influenced the submitted work.

\section{References}

1. Gaieski DF, Edwards JM, Kallan MJ, Carr BG: Benchmarking the incidence and mortality of severe sepsis in the United States. Crit Care Med. 2013, 41:1167-1174. 10.1097/CCM.0b013e31827c09f8

2. Rhodes A, Evans LE, Alhazzani W, et al.: Surviving Sepsis Campaign: international guidelines for management of sepsis and septic shock:2016. Intensive Care Med. 2017, 43:304-377. 10.1007/s00134-0174683-6 
3. Yildiz O, Doganay M, Aygen B, Guven M, Kelestimur F, Tutus A: Physiological-dose steroid therapy in sepsis [ISRCTN36253388]. Crit Care. 2002, 6:251. 10.1186/cc1498

4. Bollaert PE, Charpentier C, Levy B, Debouverie M, Audibert G, Larcan A: Reversal of late septic shock with supraphysiologic doses of hydrocortisone. Crit Care Med. 1998, 26:645-650. 10.1097/00003246-19980400000010

5. Schumer W: Steroids in the treatment of clinical septic shock . Ann Surg. 1976, 184:333-341.

6. Sprung CL, Caralis PV, Marcial EH, et al.: The effects of high-dose corticosteroids in patients with septic shock. A prospective, controlled study. New Eng J Med. 1984, 311:1137-1143. 10.1056/NEJM198411013111801

7. Lefering R, Neugebauer EA: Steroid controversy in sepsis and septic shock: a meta-analysis . Crit Care Med. 1995, 23:1294-1303. 10.1097/00003246-199507000-00021

8. Cronin L, Cook DJ, Carlet J, Heyland DK, King D, Lansang MA, Fisher CJ Jr: Corticosteroid treatment for sepsis: a critical appraisal and meta-analysis of the literature. Crit Care Med. 1995, 23:1430-1439.

9. Annane D, Sebille V, Charpentier C, et al.: Effect of treatment with low doses of hydrocortisone and fludrocortisone on mortality in patients with septic shock. JAMA. 2002, 288:862-871. 10.1001/jama.288.7.862

10. Volbeda M, Wetterslev J, Gluud C, Zijlstra JG, van der Horst IC, Keus F: Glucocorticosteroids for sepsis: systematic review with meta-analysis and trial sequential analysis. Intensive Care Med. 2015, 41:1220-1234. 10.1007/s00134-015-3899-6

11. Lv QQ, Gu XH, Chen QH, Yu JQ, Zheng RQ: Early initiation of low-dose hydrocortisone treatment for septic shock in adults: A randomized clinical trial. The. Am J Emerg Med. 2017, 35:1810-1814. 10.1016/j.ajem.2017.06.004

12. Keh D, Trips E, Marx G, et al.: Effect of hydrocortisone on development of shock among patients with severe sepsis: the HYPRESS randomized clinical trial. JAMA. 2016, 316:1775-1785. 10.1001/jama.2016.14799

13. Venkatesh B, Finfer S, Cohen J, et al.: Adjunctive glucocorticoid therapy in patients with septic shock . New Eng J Med. 2018, 378:797-808. 10.1056/NEJMoa1705835

14. Annane D, Renault A, Brun-Buisson C, et al.: Hydrocortisone plus fludrocortisone for adults with septic shock. New Eng J Med. 2018, 378:809-818. 10.1056/NEJMoa1705716

15. Suffredini AF: A role for hydrocortisone therapy in septic shock? . New Eng J Med. 2018, 378:860-861. 10.1056/NEJMe1801463

16. Liberati A, Altman DG, Tetzlaff J, Mulrow C, Gotzsche PC, Ioannidis JP: The PRISMA statement for reporting systematic reviews and meta-analyses of studies that evaluate healthcare interventions: explanation and elaboration. BMJ. 2009, 339:2700. 10.1136/bmj.b2700

17. Sprung CL, Annane D, Keh D, et al.: Hydrocortisone therapy for patients with septic shock . New Eng J Med. 2008, 358:111-124. 10.1056/NEJMoa071366

18. Higgins JP, Altman DG, Gotzsche PC, et al.: The Cochrane Collaboration's tool for assessing risk of bias in randomised trials. BMJ. 2011, 343:5928. 10.1136/bmj.d5928

19. Cochrane Handbook of Systematic Reviews of Interventions 5.1.0 Part 2: general methods for Cochrane Reviews. The Cochrane Collaboration 2011. (2011). https://training.cochrane.org/handbook.

20. Minneci PC, Deans KJ, Banks SM, Eichacker PQ, Natanson C: Meta-analysis: the effect of steroids on survival and shock during sepsis depends on the dose. Ann Intern Med. 2004, 141:47-56. 10.7326/0003-4819-141-1200407060-00014

21. Katsenos CS, Antonopoulou AN, Apostolidou EN, Ioakeimidou A, Kalpakou GT, Papanikolaou MN: Early administration of hydrocortisone replacement after the advent of septic shock: impact on survival and immune response*. Crit Care Med. 2014, 42:1651-1657. 10.1097/CCM.0000000000000318

22. Dellinger RP, Levy MM, Carlet JM, et al.: Surviving Sepsis Campaign: international guidelines for management of severe sepsis and septic shock: 2008. Crit Care Med. 2008, 36:296-327. 10.1007/s00134-0070934-2

23. Dellinger RP, Carlet JM, Masur H, et al.: Surviving Sepsis Campaign guidelines for management of severe sepsis and septic shock. Crit Care Med. 2004, 32:858-873. 10.1007/s00134-004-2210-z

24. Dellinger RP, Levy MM, Rhodes A, et al.: Surviving sepsis campaign: international guidelines for management of severe sepsis and septic shock: 2012. Crit Care Med. 2013, 41:580-637. 10.1097/CCM.0b013e31827e83af

25. Marik PE, Pastores SM, Annane D, et al.: Recommendations for the diagnosis and management of corticosteroid insufficiency in critically ill adult patients: consensus statements from an international task force by the American College of Critical Care Medicine. Crit Care Med. 2008, 36:1937-1949. 10.1097/CCM.0b013e31817603ba 